\title{
AVALIAÇÃO NEUROFUNCIONAL EM PACIENTES COM HANSENÍASE
}

\author{
Neurofunctional evaluation in patients affected by leprosy \\ Evaluación neurofuncional de pacientes con hanseniasis
}

Artigo Original

\begin{abstract}
RESUMO
Objetivo: Investigar as alterações neurofuncionais apresentadas pelos pacientes com hanseníase, buscando também identificar seu perfil socioeconômico e clínico. Métodos: Estudo transversal com 51 pacientes adultos diagnosticados com hanseníase, independentemente do sexo, realizado em centro de referência, em 2010, no qual se aplicou a ficha de "avaliação neurológica simplificada" e um questionário (dados socioeconômicos e clínicos). Achados apresentados de forma descritiva. Resultados: Encontrou-se média de idade de 46,4 $\pm 14,9$ anos, $32(62,7 \%)$ pacientes do sexo masculino, $32(62,7 \%)$ com ensino fundamental incompleto e 37 (72,5\%) com renda familiar de 1 a 3 salários mínimos. O tempo médio em tratamento era de 14,4 $\pm 15,63$ meses. Dominou a hanseníase do tipo multibacilar $(n=18 / 35,3 \%)$ e a forma tuberculoide $(n=11 / 21,6 \%)$. Articulações interfalangeanas em membros superiores e inferiores comprometidas em $5(9,8 \%)$ e $6(11,7 \%)$ pacientes, respectivamente. Nervos mais acometidos: tibial posterior em 19 (37,3\%), ulnar em 17 $(33,3 \%)$ e fibular comum em $13(25,5 \%)$ pacientes. Músculos com déficits: extensor do hálux $(n=8 / 15,7 \%)$, extensor dos artelhos $(n=6 / 11,8 \%)$ e abdutor do $5^{\circ}$ dedo $(n=6 ; 11,8 \%)$. Observou-se que $35(68,6 \%)$ pacientes tinham alterações sensitivas em membros inferiores e 14 (27,5\%) apresentavam incapacidade funcional grau 1. Conclusão: O estudo evidenciou o perfil socioeconômico dos pacientes com hanseníase como sendo homens, de baixa escolaridade e renda, com classificação operacional multibacilar apresentando a forma clínica tuberculoide. $\mathrm{Na}$ avaliação neurofuncional, houve maior ocorrência de alterações sensitivas sobre as motoras, como também discreta presença de deformidades e elevado grau de incapacidade funcional.
\end{abstract}

Descritores: Hanseníase; Perfil de Saúde; Avaliação da Deficiência; Reabilitação.

\section{ABSTRACT}

Objective: To investigate neurofunctional alterations in patients affected by leprosy, also searching to identify their socioeconomic and clinical profile. Methods: Cross-sectional study with 51 adult patients diagnosed with leprosy, regardless of gender, conducted in a reference center in 2010, in which the simplified neurofunctional evaluation form and a questionnaire (socioeconomic and clinical data) were applied. Findings were presented in a descriptive approach. Results: The study found a mean age of $46.4 \pm 14.9$ years, 32 $(62.7 \%)$ patients of male sex, 32 (62.7\%) with incomplete elementary school and $37(72.5 \%)$ with family income between 1 and 3 minimum wages. The mean time of treatment was $14.4 \pm 15.63$ months. The multibacillary type of leprosy predominated ( $n=18 / 35.3 \%)$ with tuberculoid form $(n=11 / 21.6 \%)$. The interphalangeal joints of upper and lower limbs were compromised in $5(9.8 \%)$ and $6(11.7 \%)$ patients, respectively. The most affected nerves were the posterior tibial in 19 (37.3\%), the ulnar in 17 (33.3\%), and the common fibular in 13 $(25.5 \%)$ patients. The muscles with deficit were the extensor hallucis $(n=8 / 15.7 \%)$, the toe extensors $(n=6 / 11.8 \%)$ and the 5 th finger abductor $(n=6 / 11.8 \%)$. It was observed that 35 patients (68.6\%) presented sensitive alterations in lower limbs and 14 (27.5\%) presented functional incapacity degree 1. Conclusion: The study highlighted the socioeconomic profile of leprosy patients as men, low educational level and income, with multibacillary operational classification showing tuberculoid clinical form. In the neurofunctional assessment, there were sensory changes with higher incidence than the motor alterations, as well as discreet presence of deformities and high degree of functional disability.

Descriptors: Leprosy; Health Profile; Disability Evaluation; Rehabilitation.

\author{
Rafael Mesquita ${ }^{(1)}$ \\ Luana Torres Monteiro Melo ${ }^{(2)}$ \\ Renata dos Santos Vasconcelos ${ }^{(3)}$ \\ Débora Militão Soares ${ }^{(2)}$ \\ George André Araújo Félix ${ }^{(2)}$ \\ Liana Parente de Azevedo \\ Férrer $^{(2)}$
}

Ana Paula Vasconcellos Abdon ${ }^{(2)}$

1) Programa Associado Universidade Estadual de Londrina / Universidade Norte do Paraná - UEL / UNOPAR - Londrina

(PR) - Brasil

2) Universidade de Fortaleza - UNIFOR Fortaleza (CE) - Brasil

3) Universidade Federal do Ceará - UFC Fortaleza (CE) - Brasil
Data de recebimento: $17 / 02 / 2014$ Data de revisão: 03/03/2014 Data de aceite: $12 / 04 / 2014$ 


\section{RESUMEN}

Objetivo: Investigar las alteraciones neurofuncionales presentadas por los pacientes con hanseniasis e identificar su perfil socioeconómico y clínico. Métodos: Estudio transversal con 51 pacientes adultos con el diagnóstico de hanseniasis independiente del sexo realizado en un centro de referencia en el 2010 en el cual se aplico la ficha de "evaluación neurológica simplificada" y un cuestionario (datos socioeconómicos y clínicos). Los hallazgos fueron presentados de forma descriptiva. Resultados: Se encontró una media de edad de 46,4 $\pm 14,9$ años, 32 (62,7\%) pacientes del sexo masculino, 32 (62,7\%) con enseñanza fundamental incompleta y 37 (72,5\%) con renta familiar de 1 a 3 ingresos mínimos. El tiempo medio de tratamiento fue de 14,4 \pm 15,63 meses. Hubo predominio de la hanseniasis del tipo multibacilar $(n=18 / 35,3 \%$ ) y la forma tuberculoide $(n=11 / 21,6 \%)$. Las articulaciones interfalángicas en los miembros superiores e inferiores estaban comprometidas en 5 (9,8\%) y 6 (11,7\%) pacientes, respectivamente. Los nervios más acometidos: tibial posterior en 19 (37,3\%), cubital en 17 (33,3\%) y fibular común en $13(25,5 \%)$ pacientes. Los muslos con déficits: extensor del halux $(n=8 / 15,7 \%)$, extensor de los artellos $(n=6 / 11,8 \%)$ y el abductor del $5^{\circ}$ dedo $(n=6 ; 11,8 \%)$. Se observó que $35(68,6 \%)$ tenian alteraciones sensitivas en los miembros inferiores $y$ 14 (27,5\%) presentaban incapacidad funcional de grado 1 . Conclusión: El estudio evidencio el perfil socioeconómico de los pacientes con hanseniasis de hombres, con baja escolaridad $y$ renta, de clasificación operacional multibacilar presentando la forma clínica tuberculoide. En la evaluación neurofuncional hubo más ocurrencia de alteraciones sensitivas que motoras y también discreta presencia de deformidades y elevado grado de incapacidad funcional.

Descriptores: Lepra; Perfil de Salud; Evaluación de la Discapacidad; Rehabilitación.

\section{INTRODUÇÃO}

A hanseníase é definida como uma doença crônica infectocontagiosa e curável, de evolução lenta e que afeta primariamente a pele e o sistema nervoso periférico. É causada pelo Mycobacterium leprae, que possui alta infectividade, baixa patogenicidade e é transmitido de pessoa a pessoa pelo convívio diário com doentes contagiosos sem tratamento ${ }^{(1)}$.

Segundo a Organização Mundial da Saúde (OMS), em todo o mundo, mais de 230 mil novos casos de hanseníase são identificados e cerca de 15 mil pessoas desenvolvem sequelas e/ou deformidades provocadas pela doença ${ }^{(2)}$. O Brasil é o segundo país do mundo com maior número de casos de hanseníase, sendo responsável por 34 mil novos casos. Isso significa uma taxa de detecção de 17,6 por 100 mil habitantes, e desse total, 5,4\% são em menores de 15 anos e $7,1 \%$, diagnosticados tardiamente com presença de deformidades ${ }^{(3)}$.

Embora o Brasil registre decréscimos contínuos nos coeficientes de prevalência e detecção de casos novos de hanseníase, as regiões Norte e Nordeste apresentam as mais altas taxas de prevalência, concentrando a maior parte dos casos. De acordo com o DATASUS, no Ceará, essa taxa está acima da média nacional, apresentando em 2012 um total de 2.066 casos notificados, respondendo por uma taxa de detecção de 24,0/100.000 habitantes, tendo uma população total de 8.547.750 atingidos pela doença ${ }^{(4)}$.

Ao atingir uma população economicamente ativa entre 20 e 59 anos, essa patologia repercute social e economicamente na vida das pessoas atingidas, constituindo, portanto, um importante problema de saúde pública. Assim, programas de eliminação dessa doença estão entre as ações prioritárias do Ministério da Saúde ${ }^{(5)}$.

Ela possui um grande espectro de apresentações clínicas, e o seu diagnóstico baseia-se principalmente na presença de lesões na pele, perda de sensibilidade tátil e espessamento neural. O diagnóstico correto e o tratamento adequado e precoce das reações são de grande valor para a prevenção de incapacidades, principalmente para evitar o dano neural e as incapacidades funcionais ${ }^{(1,3,6)}$.

A avaliação neurofuncional do paciente tem como objetivo pesquisar possíveis alterações neurológicas, motoras e dermatológicas provocadas pela hanseníase, sendo, portanto, imprescindível que toda a equipe de saúde seja corretamente treinada para a realização de uma avaliação eficaz, seguindo o mesmo padrão, com o foco na deteç̧ão precoce da doença, bem como na prevenção das incapacidades ${ }^{(6,7)}$.

A hanseníase é um grande problema de saúde pública. Sua detecção precoce e controle dependem diretamente da participação integrada dos sistemas municipal, estadual e federal de saúde, desde as unidades básicas de saúde aos centros especializados. É importante que sejam realizados planejamentos de ações pela equipe de saúde, direcionadas à orientação do autocuidado e à prevenção das limitações funcionais dos pacientes na realização das suas atividades cotidianas, promovendo a reabilitação ${ }^{(7,8,9)}$.

Diante do exposto, buscou-se investigar as alterações neurofuncionais apresentadas pelos pacientes com hanseníase, buscando também identificar seu perfil socioeconômico e clínico.

\section{MÉTODOS}

Estudo quantitativo do tipo transversal realizado no Centro de Referência Nacional em Dermatologia Sanitária Dona Libânia, localizado na cidade de Fortaleza-CE, no período de novembro a dezembro de 2010. 
Participaram da pesquisa 51 pacientes adultos ou idosos, com idade superior a 18 anos, independentemente do sexo, com diagnóstico clínico de hanseníase estabelecido pelos dermatologistas do serviço. Adotou-se como critério de inclusão os pacientes encaminhados para o setor de prevenção de incapacidades (PI), excluindo-se pacientes que estivessem sem diagnóstico clínico definido, pois, no serviço supracitado, os pacientes podem ser avaliados no PI antes mesmo da definição do diagnóstico de hanseníase.

Realizaram-se as avaliações duas vezes por semana, durante dois meses (novembro e dezembro), nos períodos da manhã e tarde, por um único pesquisador previamente treinado, visando reduzir erros e viés na coleta dos dados.

Para a avaliação neurofuncional, utilizaram-se o prontuário e a avaliação neurológica simplificada do Ministério da Saúde, adotada pelo Centro de Dermatologia Dona Libânia ${ }^{(10)}$, com algumas modificações necessárias ao presente estudo. Foram avaliados a sensibilidade tátil, a força muscular periférica, a presença de deformidades nas articulações interfalangeanas, o espessamento dos nervos e o grau de incapacidade. Utilizou-se também um questionário, elaborado pelos pesquisadores, para a coleta de dados socioeconômicos e clínicos ${ }^{(11)}$.

Avaliou-se a sensibilidade tátil por meio dos monofilamentos de Semmes-Weinstein ou estesiômetro de diferentes cores (verde, azul, violeta, vermelho e laranja) nas faces dorsal e plantar dos pés, dorsal e palmar das mãos, obedecendo ao trajeto dos nervos responsáveis pela sensibilidade cutânea dessas regiões. A utilização dos monofilamentos possibilita graduar a sensibilidade em vários níveis, de normal até a perda da sensibilidade profunda. Dessa forma, produz estímulos de diferentes intensidades, o que permite quantificar e monitorar a melhora/piora ou estabilidade da função neural ${ }^{(12)}$.

A mensuração da força muscular dos abdutores do $2^{\circ} \mathrm{e}$ $5^{\circ}$ dedos; intrínsecos; extensores do punho, polegar, hálux e artelhos; dorsiflexores e eversores foi realizada através da tabela de Oxford. Essa tabela é composta por 5 graus, em que o grau 0 (zero) representa a ausência de esboço de contração muscular à palpação; no grau 1, há apenas um esboço de contração muscular à palpação; no grau 2, o indivíduo consegue realizar o movimento ativo quando há eliminação da gravidade; no grau 3 , ocorre movimento ativo contra a gravidade; no grau 4 , há movimento contra a gravidade e resistência manual moderada do avaliador; no grau 5, ocorre movimento contra a gravidade e com resistência máxima do avaliador ${ }^{(13,14)}$. Considerou-se como "normal" os graus 5 ou 4, e como "déficit", os graus 3, 2 ou $1^{(13)}$.

Através de inspeção, verificou-se a presença de garras móvel ou fixa nas articulações interfalangeanas. Presença de dor, espessamento, choque, fibrose ou nódulo foram investigados através de palpação nos nervos ulnar, mediano, radial, radial cutâneo, fibular comum e tibial posterior ${ }^{(10)}$.

A classificação do grau de incapacidade física seguiu as normas do Ministério da Saúde, utilizando os seguintes critérios: grau 0 (zero), quando não há comprometimento neural nas mãos ou pés; grau 1, corresponde à diminuição ou perda de sensibilidade; grau 2, indica a presença de incapacidades e deformidades do tipo garras, reabsorção óssea, mãos e pés caídos, entre outras ${ }^{(14)}$.

Após a coleta dos dados, os resultados foram apresentados de forma descritiva, expressos em média \pm desvio padrão ou frequência absoluta e relativa. Utilizouse o programa SPSS versão 16.0 (SPSS Inc., Chicago, IL, EUA).

Este estudo teve aprovação pelo Comitê de Ética em Pesquisa da instituição, em obediência à Resolução 196/96 do Conselho Nacional de Saúde, que regulamenta os aspectos ético-legais da pesquisa em seres humanos, com parecer de $\mathrm{n}^{\circ} 008 / 08$. Todos os pacientes foram instruídos sobre a pesquisa e assinaram o Termo de Consentimento Livre e Esclarecido.

\section{RESULTADOS}

Dos 51 pacientes da amostra, 32 (62,7\%) eram do sexo masculino. A idade dos indivíduos variou entre 18 e 79 anos, com média de 46,4 $\pm 14,90$ anos.

Ao analisar o grau de escolaridade, foi visto que 32 pacientes $(62,7 \%)$ possuíam ensino fundamental incompleto; 4 (7,8\%), ensino fundamental completo; 3 $(5,9 \%)$, ensino médio incompleto; $11(21,6 \%)$, ensino médio completo; e apenas $1(2 \%)$ possuía ensino superior.

Com relação à renda familiar, 10 pacientes $(19,6 \%)$ relataram ter renda abaixo de 1 salário mínimo; 37 (72,5\%), de 1 a 3 salários mínimos; 3 (5,9\%), de 4 a 6 salários mínimos; e somente 1 (2\%) possuía renda acima de 7 salários mínimos.

Quanto à classificação operacional, encontraramse $18(35,3 \%)$ pacientes com a classificação operacional multibacilar, $8(15,7 \%)$ do tipo paucibacilares e $25(49,0 \%)$ não possuíam essa informação em seus prontuários. Sobre a apresentação clínica da hanseníase, 11 pacientes $(21,6 \%)$ apresentaram a forma tuberculoide; $10(19,6 \%)$, a forma dimorfa; e $9(17,6 \%)$, a forma virchowiana. Essas informações não estavam disponíveis para o restante dos pacientes $(n=21 / 41,2 \%)$. O tempo em tratamento no centro de referência variou de 1 dia a 72 meses, com média de 14,4 $\pm 15,63$ meses.

Do total, 46 pacientes $(90,2 \%)$ relataram estar em tratamento medicamentoso para a hanseníase. Destes, 
Tabela I - Avaliação da presença de alteração nos nervos periféricos em pacientes com hanseníase em tratamento em centro de referência de dermatologia. Fortaleza-CE, 2010.

\begin{tabular}{|c|c|c|c|}
\hline \multirow{2}{*}{ Avaliação neural } & & \multicolumn{2}{|c|}{ Achados } \\
\hline & & $\mathrm{n}$ & $\%$ \\
\hline \multirow{5}{*}{ nervo ulnar } & \multirow{5}{*}{$\begin{array}{l}\text { normal } \\
\text { tinel/choque } \\
\text { dor } \\
\text { espessamento } \\
\text { total }\end{array}$} & 34 & $66,7 \%$ \\
\hline & & 11 & $21,6 \%$ \\
\hline & & 0 & $0 \%$ \\
\hline & & 6 & $11,8 \%$ \\
\hline & & 51 & $100 \%$ \\
\hline \multirow{5}{*}{ nervo mediano } & \multirow{5}{*}{$\begin{array}{l}\text { normal } \\
\text { tinel/choque } \\
\text { dor } \\
\text { espessamento } \\
\text { total }\end{array}$} & 41 & $80,4 \%$ \\
\hline & & 10 & $19,6 \%$ \\
\hline & & 0 & $0 \%$ \\
\hline & & 0 & $0 \%$ \\
\hline & & 51 & $100 \%$ \\
\hline \multirow{5}{*}{ nervo radial } & \multirow{5}{*}{$\begin{array}{l}\text { normal } \\
\text { tinel/choque } \\
\text { dor } \\
\text { espessamento } \\
\text { total }\end{array}$} & 48 & $94,1 \%$ \\
\hline & & 2 & $3,9 \%$ \\
\hline & & 1 & $2 \%$ \\
\hline & & 0 & $0 \%$ \\
\hline & & 51 & $100 \%$ \\
\hline \multirow{5}{*}{ nervo radial cutâneo } & \multirow{5}{*}{$\begin{array}{l}\text { normal } \\
\text { tinel/choque } \\
\text { dor } \\
\text { espessamento } \\
\text { total }\end{array}$} & 48 & $94,1 \%$ \\
\hline & & 3 & $5,9 \%$ \\
\hline & & 0 & $0 \%$ \\
\hline & & 0 & $0 \%$ \\
\hline & & 51 & $100 \%$ \\
\hline \multirow{5}{*}{ nervo tibial posterior } & \multirow{5}{*}{$\begin{array}{l}\text { normal } \\
\text { tinel/choque } \\
\text { dor } \\
\text { espessamento } \\
\text { total }\end{array}$} & 32 & $62,7 \%$ \\
\hline & & 15 & $29,4 \%$ \\
\hline & & 1 & $2 \%$ \\
\hline & & 3 & $5,9 \%$ \\
\hline & & 51 & $100 \%$ \\
\hline \multirow{5}{*}{ nervo fibular comum } & normal & 38 & $74,5 \%$ \\
\hline & tinel/choque & 11 & $21,6 \%$ \\
\hline & dor & 1 & $2 \%$ \\
\hline & espessamento & 1 & $2 \%$ \\
\hline & total & 51 & $100 \%$ \\
\hline
\end{tabular}

$7(13,7 \%)$ encontravam-se também em tratamento fisioterápico, $3(4,9 \%)$ recebiam atenção da enfermagem e $1(2 \%)$ fazia terapia ocupacional, todos relacionados à doença. No momento da avaliação, 5 pacientes $(9,8 \%)$ não faziam uso de nenhum tratamento, por motivo de alta ou por ser a primeira avaliação no setor de PI.
Quanto à queixa principal, 30 pacientes $(58,8 \%)$ referiram dormência em membros superiores ou inferiores e $21(41,2 \%)$ referiram dor, edema e choque.

$\mathrm{Na}$ avaliação funcional, detectou-se que 5 pacientes $(9,8 \%)$ tinham alteração nas articulações interfalangeanas dos membros superiores, sendo em 3 pacientes $(5,9 \%)$ 
do tipo garra móvel e em 2 (3,9\%) do tipo garra fixa. Em membros inferiores, 6 pacientes $(11,7 \%)$ apresentaram alterações, sendo em 4 pacientes (7,8\%), garra móvel e em $2(3,9 \%)$, garra fixa. Os outros 40 pacientes $(78,5 \%)$ não apresentaram alterações nessas articulações.

Ao avaliar os nervos dos membros superiores, verificouse que 17 pacientes $(33,3 \%)$ apresentaram alteração no ulnar; 10 (19,6\%), no mediano; 3 (5,9\%), no radial; e 3 (5,9\%), no nervo radial cutâneo. Nas lesões neurais, o tipo mais encontrado foi o tinel/choque (Tabela I).

As alterações neurais de membros inferiores constaram de alteração no nervo tibial posterior em 19 pacientes $(37,3 \%)$, e $13(25,5 \%)$ apresentaram lesão no nervo fibular comum. O tipo de lesão mais detectada também foi tinel/ choque (Tabela I).
Ao avaliar a força dos músculos do membro superior, 6 pacientes $(11,8 \%)$ apresentaram déficit no abdutor do $5^{\circ}$ dedo; 5 (9,8\%), nos músculos intrínsecos; 4 (7,8\%), no abdutor do $2^{\circ}$ dedo; 2 (3,9\%), nos extensores do polegar; e nenhum nos extensores do punho (Tabela II).

Nos músculos dos membros inferiores, 8 pacientes $(15,7 \%)$ tinham déficit no músculo extensor do hálux; 6 (11,6\%), nos músculos extensores dos artelhos; 2 (3,9\%), no músculo dorsoflexor; e 2 (3,9\%), nos músculos eversores de tornozelo (Tabela III).

Relativo à sensibilidade superficial, 7 pacientes $(13,7 \%)$ apresentaram alteração nos membros superiores e $35(68,6 \%)$, em membros inferiores.

Na classificação do grau de incapacidade, encontraramse $26(51,0 \%)$ pacientes normais, $14(27,5 \%)$ com grau 1 , e $11(21,6 \%)$ com grau 2.

Tabela II - Avaliação da força muscular nos membros superiores em pacientes com hanseníase em tratamento em centro de referência de dermatologia. Fortaleza-CE, 2010.

\begin{tabular}{|c|c|c|c|}
\hline \multirow{2}{*}{ Avaliação muscular } & & \multicolumn{2}{|c|}{ Achados } \\
\hline & & $\mathrm{n}$ & $\%$ \\
\hline abdutor do $5^{\circ}$ dedo & $\begin{array}{l}\text { normal } \\
\text { bom } \\
\text { regular } \\
\text { fraco } \\
\text { insuficiente }\end{array}$ & $\begin{array}{c}43 \\
2 \\
3 \\
2 \\
1\end{array}$ & $\begin{array}{c}84,3 \% \\
3,9 \% \\
5,9 \% \\
3,9 \% \\
2 \%\end{array}$ \\
\hline intrínsecos & $\begin{array}{l}\text { normal } \\
\text { bom } \\
\text { regular } \\
\text { fraco } \\
\text { insuficiente }\end{array}$ & $\begin{array}{c}43 \\
3 \\
1 \\
3 \\
1\end{array}$ & $\begin{array}{c}84,3 \% \\
5,9 \% \\
2 \% \\
5,9 \% \\
2 \%\end{array}$ \\
\hline abdutor do $2^{\circ}$ dedo & $\begin{array}{l}\text { normal } \\
\text { bom } \\
\text { regular } \\
\text { fraco } \\
\text { insuficiente }\end{array}$ & $\begin{array}{c}46 \\
1 \\
1 \\
2 \\
1\end{array}$ & $\begin{array}{c}90,2 \% \\
2 \% \\
2 \% \\
3,9 \% \\
2 \%\end{array}$ \\
\hline extensores do polegar & $\begin{array}{c}\text { normal } \\
\text { bom } \\
\text { regular } \\
\text { fraco } \\
\text { insuficiente }\end{array}$ & $\begin{array}{c}49 \\
0 \\
1 \\
0 \\
1\end{array}$ & $\begin{array}{c}96,1 \% \\
0 \% \\
2 \% \\
0 \% \\
2 \%\end{array}$ \\
\hline extensores do punho & $\begin{array}{c}\text { normal } \\
\text { bom } \\
\text { regular } \\
\text { fraco } \\
\text { insuficiente }\end{array}$ & $\begin{array}{c}51 \\
0 \\
0 \\
0 \\
0\end{array}$ & $\begin{array}{c}100 \% \\
0 \% \\
0 \% \\
0 \% \\
0 \%\end{array}$ \\
\hline
\end{tabular}


Tabela III - Avaliação da força muscular nos membros inferiores em pacientes com hanseníase em tratamento em centro de referência de dermatologia. Fortaleza-CE, 2010.

\begin{tabular}{lccc}
\hline \multirow{2}{*}{ Avaliação muscular } & & Achados & \% \\
\cline { 2 - 3 } & & $\mathbf{n}$ & $84,3 \%$ \\
extensor do hálux & normal & 43 & $0 \%$ \\
& bom & 0 & $5,9 \%$ \\
& regular & 3 & $2 \%$ \\
extensores dos artelhos & insuficiente & 1 & $7,8 \%$ \\
& normal & 4 & $84,3 \%$ \\
dorsoflexor & bom & 43 & $3,9 \%$ \\
& regular & 2 & $2 \%$ \\
& fraco & 1 & $2 \%$ \\
& insuficiente & 1 & $7,8 \%$ \\
eversores & normal & 4 & $94,1 \%$ \\
& bom & 48 & $2 \%$ \\
& regular & 1 & $0 \%$ \\
& fraco & 0 & $2 \%$ \\
& insuficiente & 1 & $2 \%$ \\
\hline
\end{tabular}

\section{DISCUSSÃO}

Dos 51 pacientes estudados na presente pesquisa, verificou-se que a maioria era do sexo masculino. Esse achado corrobora com a literatura, segundo a qual grande parte das doenças transmissíveis predomina nesse $\operatorname{sexo}^{(6)}$. Além disso, encontra-se também que a hanseníase em adultos é mais frequente no sexo masculino, e o risco de exposição é determinante dessa diferença ${ }^{(15)}$.

Com relação à faixa etária, a média nesta atual pesquisa foi de 46,4 anos. Em outros estudos, observa-se aumento expressivo e inesperado a partir dos 40 anos de idade ${ }^{(16,17)}$. Uma das possíveis explicações seria a possibilidade de uma inadequação do diagnóstico de hanseníase nessa faixa etária, uma vez que doenças poderiam levar a avaliações falso-positivas, como a diabetes mellitus ou insuficiências arteriais e venosas, principalmente na ausência de lesões clássicas da hanseníase ${ }^{(15,16)}$.
Ao analisar o grau de escolaridade e a renda familiar na amostra selecionada na presente investigação, percebeu-se que a maioria encontrava-se desfavorecida. Estudo realizado em um centro de saúde em São Paulo, com 37 sujeitos com hanseníase, mostra que $70 \%$ tinham baixa escolaridade, resultando em renda familiar precária de aproximadamente um a três salários mínimos ${ }^{(11)}$. Outro estudo, que utilizou dados do Sistema de Informação de Agravos de Notificação no período de 2001 a 2009 no estado de Minas Gerais, observou predominância do ensino fundamental completo, com renda familiar de um a três salários mínimos ${ }^{(17)}$.

Ao analisar o tempo em tratamento no centro de referência investigado no estudo em questão, pode-se observar que a média da amostra supera 12 meses. Segundo a OMS, a alta dos pacientes com hanseníase do tipo paucibacilar leva em média 6 meses (a partir da avaliação e/ou registro das incapacidades), e no tipo multibacilar, 
de 12 a 24 meses, com reavaliações a cada 6 meses $^{(18,19)}$. Assim, esse tempo de tratamento está de acordo com o número elevado do tipo multibacilar encontrado na presente pesquisa.

No Brasil, o Ministério da Saúde sugere uma classificação operacional com os seguintes critérios: paucibacilares (PB), os pacientes possuem imunidade celular preservada; baciloscopia negativa, até cinco lesões de pele e um nervo atingido; multibacilares (MB), correspondem a formas clínicas com imunidade específica ao bacilo reduzida ou ausente; baciloscopia positiva, mais de cinco lesões de pele e mais de um nervo atingido ${ }^{(3)}$.

Sobre a classificação operacional do tipo de hanseníase, verificou-se que $35,3 \%$ dos pacientes apresentavam a classificação MB na presente pesquisa. Esse dado diverge de um estudo realizado no município de Fortaleza-CE, onde foi verificada que a forma PB é a mais frequente, chegando a $67 \%$ das formas identificadas no ano de 2006. Apenas nos anos de 2003 e 2007 houve uma equivalência nessa proporção, com uma discreta superioridade no número de casos da forma $\mathrm{MB}^{(20)}$.

Um estudo ${ }^{(21)}$ verificou que, no Ceará, a forma clínica tuberculoide é a mais frequente, chegando a mais de $50 \%$ dos casos detectados no ano de 2006 e se mantendo em valores próximos a esse nos demais anos. No ano de 2007, houve uma queda nesse percentual, mas deve ser destacada a elevada proporção de casos classificados como ignorados ou em branco. A forma tuberculoide é característica em pessoas que apresentam resistência ao Mycobacterium leprae, mas sofreram contatos sucessivos e desenvolveram a doença ${ }^{(21,22)}$. Os resultados do presente estudo corroboram com esses resultados, pois obervou-se uma predominância da forma clínica tuberculoide.

Neste estudo, pôde-se verificar a importância do uso das drogas medicamentosas, pois a maioria $(n=46)$ recebia tratamento medicamentoso, contribuindo para a boa evolução dos pacientes. Estudos anteriores mostraram a eficiência do tratamento com a poliquimioterapia (PQT), com queda da taxa de deformidades entre os casos novos. Esse protocolo de tratamento tem possibilitado diagnóstico precoce e acompanhamento sistematizado associado ao monitoramento neural, intervenções para diagnóstico e tratamentos adequados (medicamentoso e não medicamentoso) dos episódios de neuropatia associados ou não a quadros reacionais ${ }^{(23,24)}$. Apesar dessas práticas, observou-se elevado índice de pacientes com incapacidades neurológicas.

Quanto à queixa principal, a maioria dos pacientes referiu dormência em membros superiores ou inferiores no presente estudo. Dentre as manifestações clínicas da hanseníase, pode-se mencionar dor intensa, hipersensibilidade do nervo, edema, déficit motor e sensitivo. No entanto, em grande parte, os fenômenos da neurite podem se desenvolver sem dor, sendo denominados de neurites silenciosas. Nelas, não se observam os achados de dor ou a hipersensibilidade do nervo, mas as alterações de sensibilidade e de força motora ocorrem e, muitas vezes, só podem ser detectadas por exame de sensibilidade e força muscular $^{(19,20)}$.

Durante a inspeção das articulações interfalangeanas, verificou-se que a maioria dos pacientes não apresentava alterações na pesquisa em questão. As deformidades e incapacidades encontradas nos membros superiores e inferiores, denominadas "garras", são devidas aos fatores relacionados ao comprometimento nervoso: as alterações de sensibilidade e da motricidade, e os estados imunoinflamatórios ${ }^{(20,21)}$.

$\mathrm{O}$ acometimento neural ocorre em todas as formas de hanseníase, sendo sua característica mais marcante e responsável pelo estigma de enfermidade deformante ${ }^{(19,25)}$. Porém, não foram observadas alterações durante a palpação dos nervos dos membros superiores e inferiores na maioria dos pacientes.

Os bacilos de Hansen atingem especialmente os nervos periféricos, desde as suas terminações da derme até os troncos nervosos. A neuropatia hanseniana é clinicamente mista, comprometendo tanto fibras nervosas sensitivas como motoras e autonômicas, resultando em alterações e desequilíbrios de flexibilidade e força ${ }^{(20,26)}$. No presente estudo, observou-se, durante a avaliação da força muscular, que a maioria dos pacientes apresentou grau 5.

$\mathrm{Na}$ presente investigação, avaliou-se o grau de incapacidade no momento da realização da avaliação no setor da PI, portanto, com a maioria dos pacientes com PQT em andamento e com os demais pacientes após a alta. A literatura relata que essa avaliação deve ser realizada nas etapas de diagnóstico da doença e na alta, sendo utilizados dados secundários ${ }^{(16,23)}$.

Como a proporção de grau de incapacidades encontrada foi considerada alta, sugere-se a necessidade de um diagnóstico mais precoce. Outros estudos também mostraram um número elevado, assim, incapacidades são reconhecidamente as principais responsáveis pelo estigma da doença, podendo prejudicar o convívio social quanto ao seu desenvolvimento ${ }^{(8,15,23)}$.

Estudos revelam a importância do diagnóstico precoce da hanseníase, assim como a necessidade de avaliação e acompanhamento na prevenção de incapacidades, tendo ambos um forte impacto na redução e inibição do aparecimento das incapacidades ${ }^{(9,16,19)}$.

Nesse contexto, é importante ressaltar a necessidade de priorizar a atenção integrada a esse paciente, com medidas de planejamento e educação continuada nas unidades 
de atenção à saúde. É imprescindível que ocorram ações inovadoras de capacitação dos profissionais envolvidos direta e indiretamente na assistência a esses pacientes, buscando a padronização de medidas de avaliação e detecção precoce, além de ações de orientação e controle de agravos, com o objetivo de prevenir incapacidades e deformidades funcionais e proporcionar um atendimento à saúde de qualidade ${ }^{(27,28)}$

O presente estudo pode contribuir para um quadro mais detalhado das incapacidades físicas decorrentes da hanseníase e, consequentemente, servir como base para traçar futuras propostas a fim de reduzir a incidência delas. Ressalta-se, entretanto, que a quantidade reduzida de pacientes participantes nesta pesquisa e a ausência de dados nos prontuários apresentam-se como limitações.

\section{CONCLUSÃO}

O estudo evidenciou o perfil socioeconômico dos pacientes com hanseníase como sendo homens, de baixa escolaridade e renda, com classificação operacional multibacilar apresentando a forma clínica tuberculoide.

$\mathrm{Na}$ avaliação neurofuncional, foi encontrada maior ocorrência de alterações sensitivas sobre as motoras, como também discreta presença de deformidades. Porém, esses achados não impediram a detecção de elevado grau de incapacidade funcional.

\section{REFERÊNCIAS}

1. Penna ML, Oliveira ML, Penna GO. The epidemiological behaviour of leprosy in Brazil. Lepr Rev. 2009;80(3):332-44.

2. World Health Organization- WHO. Enhanced global strategy for further reducing the disease burden due to leprosy (plan period: 2011-2015). New Delhi. 2011;36(86):389-400.

3. Penna GO, Domingues CMAS, Siqueira Junior JB, Elkhoury ANSM, Cechinel MP, Grossi MAF, et al. Doenças dermatológicas de notificação compulsória no Brasil. An Bras Dermatol. 2011;86(5):865-77.

4. Prefeitura Municipal de Fortaleza, Secretaria Municipal de Saúde, Célula de Vigilância Epidemiológica. Informe epidemiológico: hanseníase. Bol Saúde Fortaleza [periódico na internet]. 2011 [acesso em 2013 Set 20];15(1):1-47. Disponível em: http:// www.sms.fortaleza.ce.gov.br/images/boletins/Bol_ Hans_28112011final2.pdf

5. Oliveira VM, Assis CRD, Silva KCC. Levantamento epidemiológico da hanseníase no nordeste brasileiro durante o período de 2001-2010 Scire Salutis. 2013;3(1):16-27.

6. Miranzi SSC, Pereira LHM, Nunes AA. Perfil epidemiológico da hanseníase em um município brasileiro, no período de 2000 a 2006. Rev Soc Bras Med Trop. 2010;43(1):62-7.

7. Souza VB, Silva MRF, Silva LMS, Torres RAM, Gomes KWL, Fernandes MC, et al. Perfil epidemiológico dos casos de hanseníase de um centro de saúde da família. Rev Bras Promoç Saúde. 2013;26(1):110-6.

8. Alves CJM, Barreto JA, Fogagnolo L, Contin LA, Nassif PW. Avaliação do grau de incapacidade dos pacientes com diagnóstico de hanseníase em Serviço de Dermatologia do Estado de São Paulo. Rev Soc Bras Med Trop. 2010;43(4):460-1.

9. Rodini FCB, Gonçalves M, Barros ARSB, Mazzer N, Elui VMC, Fonseca MCR. Prevenção de incapacidade na hanseníase com apoio em um manual de autocuidado para pacientes. Fisioter Pesqui. 2010;17(2):157-66.

10. Ministério da Saúde (BR), Secretaria de Políticas da Saúde, Departamento de Atenção Básica. Guia para o controle da hanseníase. Brasília: Ministério da Saúde; 2002 .

11. Duarte MTC, Ayres JA, Simonetti JP. Perfil socioeconômico e demográfico de portadores de hanseníase atendidos em consulta de enfermagem. Rev Latinoam Enferm. 2007;15(Esp):774-9.

12. Tracey EH, Greene AJ, Doty RL. Optimizing reliability and sensitivity of Semmes-Weinstein monofilaments for establishing point tactile thresholds. Physiol Behav. 2012;105(4):982-6.

13. Hislop HJ, Montgomery J. Testes dos músculos das extremidades superiores e inferiores. In: Hislop HJ, Montgomery J. Daniels e Worthingham: provas de função muscular: técnicas de exame manual. $8^{\mathrm{a}}$ ed. São Paulo: Elsevier; 2008. p. 61-235.

14. Véras LST, Vale RGS, Mello DB, Castro JAF, Dantas EHM. Avaliação da dor em portadores de hanseníase submetidos à mobilização neural. Fisioter Pesqui. 2011;18(1):31-6.

15. Corrêa RGCF, Aquino DMC, Caldas AJM, Amaral DKCR, França FS, Mesquita ERRBPL. Epidemiological, clinical, and operational aspects of leprosy patients assisted at a referral service in the state of Maranhão, Brazil. Rev Soc Bras Med Trop. 2012;45(1):89-94.

16. Monteiro LD, Alencar CHM, Barbosa JC, Braga KP, Castro MD, Heukelbach J. Physical disabilities in 
leprosy patients after discharge from multidrug therapy in Northern Brazil. Cad Saúde Pública. 2013;29(5):90920.

17. Ribeiro Júnior AF, Vieira MA, Caldeira AP. Perfil epidemiológico da hanseníase em uma cidade endêmica no Norte de Minas Gerais. Rev Bras Clin Med. 2012;10(4):272-7.

18. Ministério da Saúde (BR). Manual de prevenção de incapacidades. $3^{\mathrm{a}}$ ed. rev. ampl. Brasília: Ministério da Saúde; 2008. (Cadernos de prevenção e reabilitação em hanseníase; n. 1).

19. Ikehara E, Nardi SMT, Ferrigno ISV, Pedro HSP, Paschoal VDA. Escala Salsa e grau de incapacidades da Organização Mundial de Saúde: avaliação da limitação de atividades e deficiência na hanseníase. Acta fisiátrica. 2010;17(4):169-74.

20. Leite VMC, Lima JWO, Gonçalves HS. Neuropatia silenciosa em portadores de hanseníase na cidade de Fortaleza, Ceará, Brasil. Cad Saúde Pública. 2011;27(4):659-65.

21. Barbosa JC, Ramos Júnior AN, Alencar MJF, Castro CGJ. Pós-alta em Hanseníase no Ceará: limitação da atividade funcional, consciência de risco e participação social. Rev Bras Enferm. 2008;61(Esp):727-33.

22. Ministério da Saúde (BR), Departamento da Atenção Básica. Programa nacional de controle de hanseníase. Brasília: Ministério da Saúde; 2008.

23. Castro RNC, Veloso TC, Matos Filho LJS, Coelho LC, Pinto LB, Castro AMNC. Avaliação do grau de incapacidade física de pacientes com hanseníase submetidos ao Dermatology Quality Life Index em Centro de Referência e Unidades Básicas de Saúde de São Luis, MA. Rev Soc Bras Clín Méd. 2009;7(6):3902.
24. Gonçalves SD, Sampaio RF, Antunes CMF. Predictive factors of disability in patients with leprosy. Rev Saúde Pública. 2009;43(2):267-74.

25. Silva Sobrinho RA, Mathias TAF, Gomes EA, Lincoln PB. Avaliação do grau de incapacidade em hanseníase: uma estratégia para sensibilização e capacitação da equipe de enfermagem. Rev Latinoam Enferm. 2007;15(6):1125-30.

26. Sociedade Brasileira de Hansenologia, Academia Brasileira de Neurologia, Sociedade Brasileira de Neurofisiologia Clínica. Hanseníase: diagnóstico e tratamento da neuropatia [internet]. São Paulo: Associação de Médica Brasileira/Conselho Federal de Medicina; 2003 [acesso em 2013 Jul 15). Disponível em: http://www.projetodiretrizes.org.br/projeto diretrizes/055.pdf

27. Costa VHMV, Cavalcanti LA, Faria-Junior JAD, Kitaoka EG, Mascarenhas GS, Mascarenhas NB, et al. Programa nacional de eliminação da hanseníase: um estudo sobre a avaliabilidade do programa e das suas ações em âmbito estadual e municipal. Rev Baiana Saúde Pública. 2010;34(3):450-67.

28. Marques M. Nova estratégia de treinamento em hanseníase para profissionais de saúde de Mato Grosso do Sul. In: Congresso Consad de Gestão Pública; 2013 Abr 16-18; Brasília. Brasília: Consad; 2013. p.1-13.

\section{Endereço para correspondência:}

Rafael Mesquita

Centro de Pesquisa em Ciências da Saúde

Universidade Norte do Paraná (UNOPAR)

Avenida Paris, 675

Bairro: Jardim Piza

CEP: 86041-120 - Londrina - Paraná - Brasil

E-mail: rafaelmesquita14@ymail.com

Instituição de origem: Universidade Norte do Paraná 